\title{
Betriebsräte fördern Lohngleichheit
}

Betriebe mit einer gewählten Interessenvertretung bezahlen mehr als 10 \% höhere Löhne als betriebsratslose Betriebe. Dieser Lohnaufschlag ist größer als derjenige für Tariflöhne. Von ihm profitieren auch die Beschäftigtengruppen, die üblicherweise auf dem Arbeitsmarkt benachteiligt sind. Damit bewirken Betriebsräte eine niedrigere Lohnungleichheit innerhalb eines Betriebes. Dies kann in Lohnschätzungen mit aktuellen und repräsentativen deutschen Daten gezeigt werden, die Merkmale der Beschäftigten mit betrieblichen Charakteristika verknüpfen. ${ }^{1}$

\section{Einleitung}

Betriebsräte haben über ihre gesetzlich verankerten umfangreichen Mitspracherechte einen direkten und nachhaltigen Einfluss auf die Arbeitsbedingungen der Beschäftigten und das wirtschaftliche Wohlergehen ihrer Betriebe. Um einen objektiven Blick auf die Bedeutung von Betriebsräten in den von ihnen mitkontrollierten Betrieben zu bekommen, ist es notwendig, repräsentative Daten zu verwenden, die sich auf vergleichbare Betriebe mit und ohne Interessenvertretung beziehen. Üblicherweise stehen der Einfluss von Betriebsräten auf Produktivität und Beschäftigungssicherheit bzw. andere Kernaktivitäten der Interessenvertretungen im Mittelpunkt des Analyseinteresses. In diesem Beitrag soll es jedoch um den Einfluss von Betriebsräten auf die betriebliche Lohnstruktur gehen, ein Thema, das aus meiner Sicht lange vernachlässigt wurde. Dies ist umso bedauerlicher, weil die Lohnstruktur bekanntermaßen eine wichtige Größe sowohl für die Beschäftigungsmöglichkeiten und die Motivation der Beschäftigten als auch für die gesamtgesellschaftliche Wohlfahrt ist.

Bisher richtete sich der Blick vornehmlich auf die Tarifverhandlungen zwischen Arbeitgeberverbänden und Gewerkschaften, wenn es darum ging, die Höhe der individuellen Löhne in Deutschland zu erklären. Dem Einfluss von Betriebsräten, der zweiten wichtigen Säule industrieller Beziehungen in Deutschland, auf Lohnhöhe und Lohnstruktur wurde jedoch kaum Aufmerksamkeit geschenkt. Dies mag zum einen daran liegen, dass Betriebsräte gemäß $§ 77$ Abs. 3 BetrVG (Betriebsverfassungsgesetz) nur mit ausdrücklicher Genehmigung der Tarifparteien direkt an ent- geltrelevanten Betriebsvereinbarungen beteiligt werden. Zum anderen fehlten bis vor kurzem geeignete Datenquellen, um das Thema empirisch zu untersuchen.

So nutzen erste vorliegende Studien zu diesem Untersuchungsgegenstand (FitzRoy/Kraft 1985; Addison et al. 1993) nur eine geringe Anzahl und möglicherweise nicht repräsentative Auswahl von Betrieben als Berechnungsbasis und kamen zu widersprüchlichen Befunden. Auch neuere Studien auf Basis des Hannoveraner Firmenpanels, das Firmen aus dem verarbeitenden Gewerbe in Niedersachsen umfasst, kommen zu unterschiedlichen Ergebnissen bezüglich einer Lohnwirkung von Betriebsräten (Addison et al. 2001; Hübler/ Jirjahn 2003). Alle diese Studien basieren auf der Lohnsumme bzw. dem Durchschnittslohn aller Beschäftigten. In Ermangelung der individuellen Lohnangaben können jedoch keine Aussagen zur Lohnstruktur gemacht werden. Erst verknüpfte Daten mit Angaben zu einzelnen Beschäftigten und Betrieben ermöglichen die Analyse des Einflusses von Betriebsräten sowohl auf die Lohnstruktur als auch auf die Lohnhöhe. Neben Papieren, die auf Basis des verknüpften Datensatzes des IAB (LIAB) oder Daten der amtlichen Statistik den Einfluss von Tarifverträgen untersuchen, ohne ausführlich auf die Wirkung von Betriebsräten einzugehen (Kölling et al. 2005; Stephan/Gerlach 2005), erhebt bisher nur die Studie von Gürtzgen (2006) den Lohneffekt von Betriebsräten. Da diese Analyse allerdings nicht im Fokus ihres Interesses liegt, werden die Befunde nicht kommentiert.

Betriebsräte haben, wie gesagt, keinen direkten Einfluss auf die tarifliche Lohngestaltung. Dennoch haben sie indirekte Einwirkungsmöglichkeiten auf die Löhne der Beschäftigten: Beispielsweise durch ihre Mitsprache bei der Klassifizierung von
Tätigkeiten und aufgrund der Möglichkeit, Vorschläge zu machen, wer befördert wird oder Weiterbildung erhält, die sich später in einer Höhergruppierung niederschlagen kann. Auch ihr Mitspracherecht bei Sonderzahlungen oder Akkordentlohnung ist relevant für die Entlohnung.

In Anlehnung an die einschlägigen Theorien der gewerkschaftlichen Lohnsetzung wäre zu vermuten, dass Betriebsräte versuchen, zum einen den Interessen der Beschäftigten an gleichen Löhnen für gleiche Tätigkeiten Rechnung zu tragen; zum anderen, dass sie sich für eine geringe Lohnspreizung einsetzen. Eine weitere Hypothese ist, dass sie vor allem Mitarbeitern zu Lohnvorteilen verhelfen, die ähnliche Eigenschaften haben bzw. in ähnlichen beruflichen Positionen sind wie sie selbst. Entlang dieser These müssten z.B. Frauen und ausländische Beschäftigte eher im Schatten des lohnrelevanten Engagements der Interessenvertretungen stehen. Denn obwohl der Anteil von Frauen und Ausländern innerhalb der gewählten Interessenvertretungen in den letzten Jahren zugenommen hat (Wassermann/Rudolph 2005), sind nach wie vor überproportional viele männliche Deutsche mit einer relativ langen Betriebszugehörigkeit und einem mittleren Berufsbildungsabschluss (Lehre oder Meister) unter den Betriebsräten vertreten.

Dieser Beitrag basiert auf Addison et al. 2006.

Thomas Zwick, PD Dr., Zentrum für Euro päische Wirtschaftsforschung (ZEW), Mannheim. Arbeitsschwerpunkte: Empirische Arbeitsmarktforschung, Industrielle Beziehungen, Personalmanagement. e-mail: zwick@zew.de 


\section{Daten}

Für unsere Analyse wurde die Welle 2001 des verknüpften Datensatzes des IAB (LIAB) verwendet, wobei nur Vollzeitbeschäftigte in Betrieben mit Gewinnmaximierungsziel einbezogen wurden. Der öffentliche Dienst wurde folglich nicht berücksichtigt. Wir haben somit Daten über gut 1,3 Mio. Beschäftigte in etwa $8.500 \mathrm{Be}-$ trieben. Die Tageslöhne sind an der Beitragsgrenze zur Sozialversicherung zensiert und deshalb imputieren wir die zensierten Werte. Hierfür wurden 20 Zellen nach Geschlecht, Ausbildung (sechs Gruppen) sowie Nationalität gebildet, und für jede Zelle wurden zensierte Lohnregressionen einzeln geschätzt - mit den Kovariaten Betriebserfahrung, Betriebserfahrung im Quadrat, Alter, Betriebssektor (16 Dummies), Ost-/West-Deutschland und drei Dummy-Variablen für das Ausbildungsniveau (Gartner 2005). Ähnlich sind wir bei den ebenfalls zensierten Variablen Betriebserfahrung und Berufserfahrung verfahren.

\section{Schätzstrategie und Ergebnisse}

Wir konzentrieren uns darauf, individuelle Lohnregressionen zu schätzen. Neben den üblicherweise in den sogenannten Mincer-Gleichungen berücksichtigten Variablen, wie Alter, Berufserfahrung, Qualifikation und Geschlecht, verwenden wir einen Indikator für das Vorhandensein eines Betriebsrats sowie weitere individuelle und betriebliche Charakteristika (Dummy für persönliche Eigenschaften bzw. für betriebliche Merkmale wie Betriebsgröße, die Bindung (oder Orientierung) an Tarifverträge(n) auf betrieblicher oder sektoraler Ebene, den Wirtschaftssektor oder die regionale Lage). In einer Schätzung für das Jahr 2001 beträgt der Lohnaufschlag für die Beschäftigten in Betrieben mit Betriebsrat gut $13 \%$ (Tabelle 1, Spalte 2).

Eine derartige Schätzspezifikation ist jedoch möglicherweise aus zwei Gründen verzerrt: Ein Schätzproblem ist, dass ein Betriebsrat nicht „zufällig“ existiert, d.h. Betriebsräte werden insbesondere in Be-

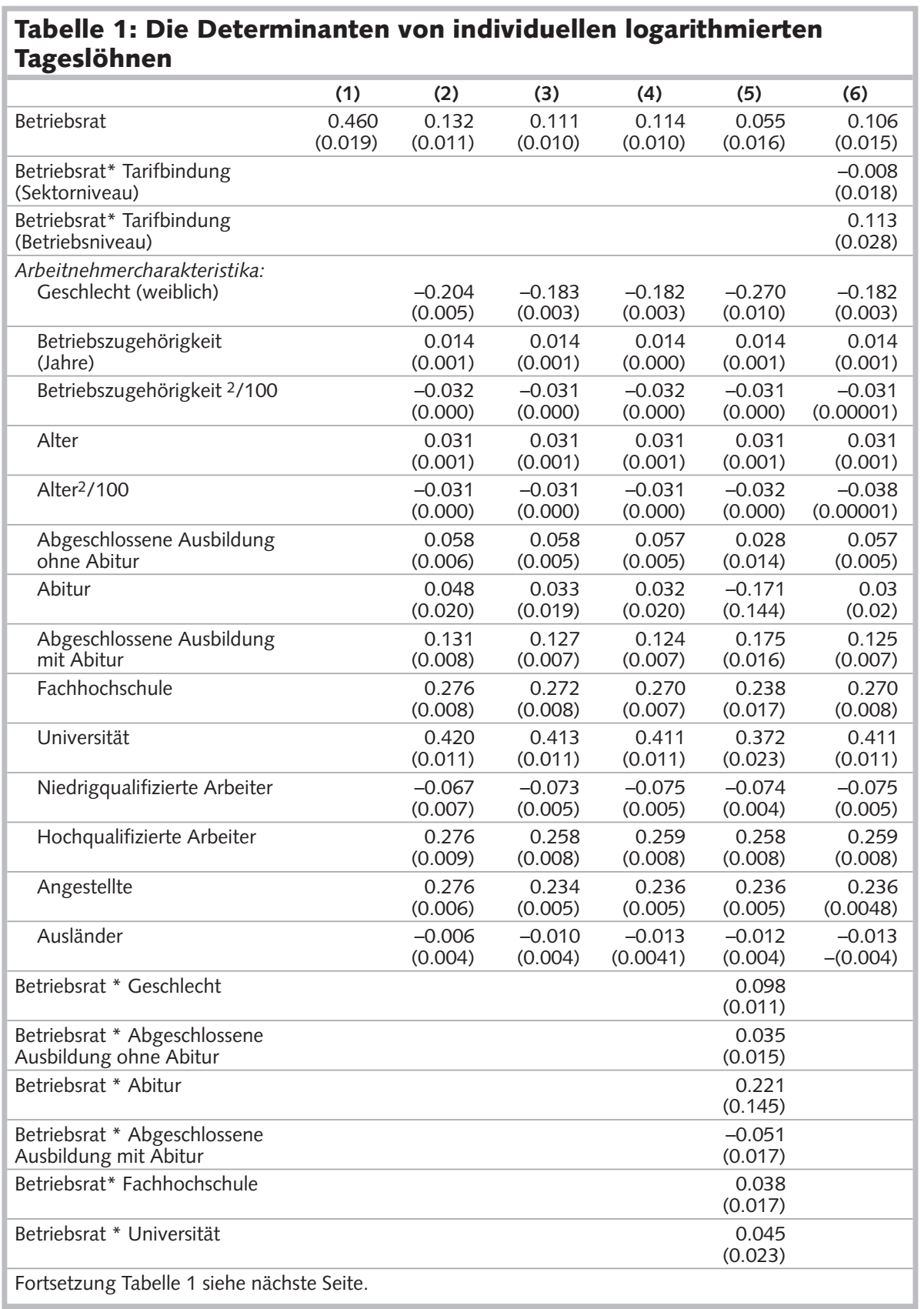

trieben gewählt, in denen sich eine Interessenvertretung aus Sicht der Beschäftigten lohnt bzw. notwendig ist. Eine Motivation für die Wahl einer Interessenvertretung könnte sein, dass der Betrieb bereits vor der Gründung eines Betriebsrates aufgrund eines höheren Gewinns oder einer höheren Produktivität als vergleichbare Betriebe höhere Löhne gezahlt hat. Mit der Konstituierung einer betrieblichen Interessenvertretung wollten die Mitarbeiter diesen Lohnvorteil absichern. Wenn man keine geeigneten Maße für die Bestimmung der Produktivität oder des Gewinns hat, läuft man Gefahr, den Lohnaufschlag fälschlicherweise der Existenz eines Betriebsrats zuzurechnen. Eine gute Möglichkeit, dieses sogenannte Endogenitätsproblem zu beseitigen, ist die Verwendung einer Selektionsgleichung, in der das Vorhandensein eines Betriebsrats erklärt wird (Heckman 1976). Anschließend wird für jeden Betrieb die vorhergesagte individuelle Wahrscheinlichkeit, einen Betriebsrat zu haben, als Korrekturvariable hinzugefügt.

Das zweite Schätzproblem ist, dass die Beschäftigten in Betrieben mit Betriebsrat „unbeobachtete“, also in der Analyse nicht berücksichtigte Eigenschaften aufweisen, die ihre individuellen Verdienstmöglichkeiten erhöhen. Denkbare Beispiele hierfür wären eine höhere Loyalität zum Betrieb, eine stärkere Ausrichtung an den wirtschaftlichen Zielen des Betriebs im tägli- 


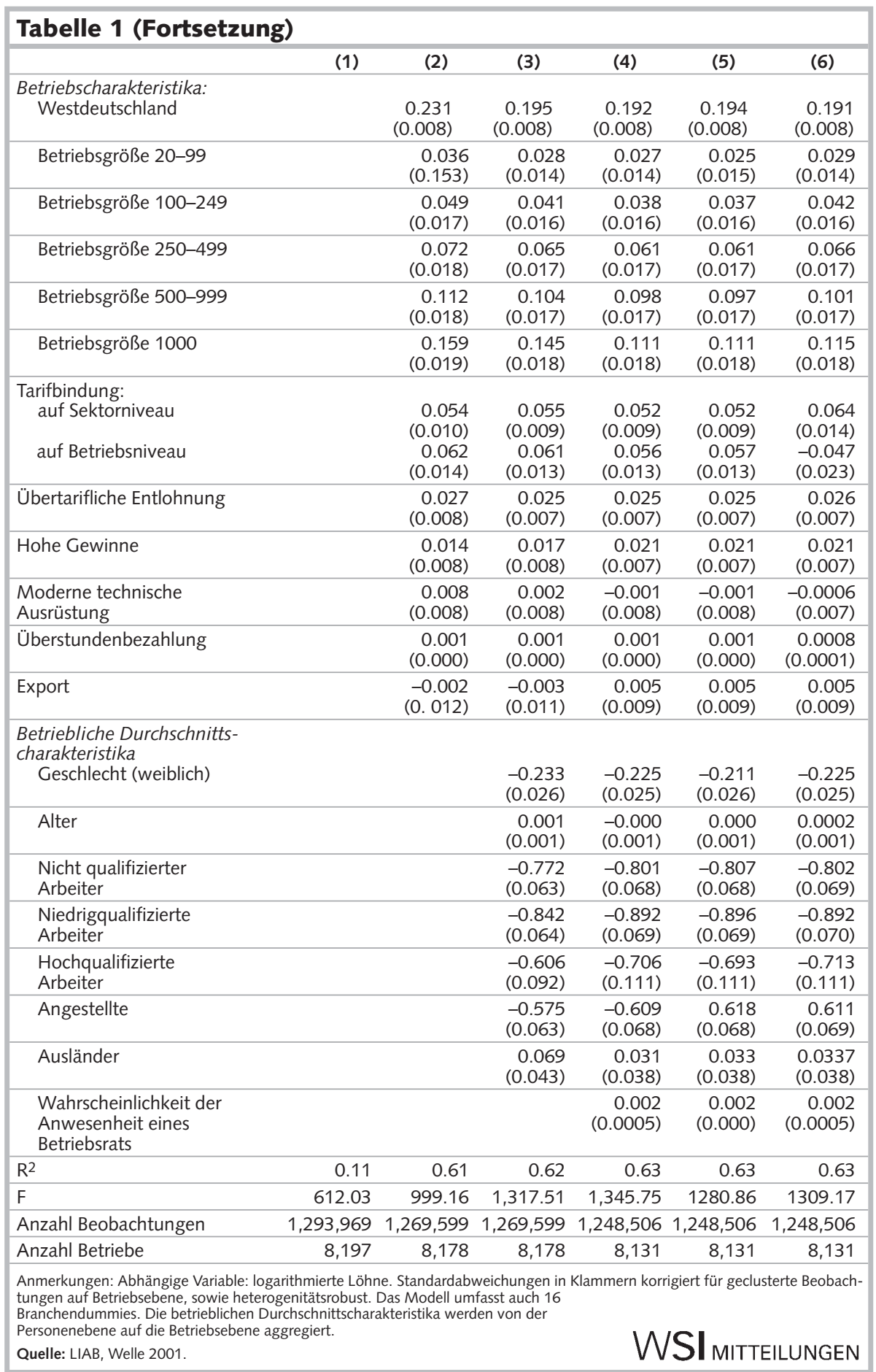

chen Handeln etc. Auch eine solche Konstellation kann fälschlicherweise dem Vorhandensein eines Betriebsrates zugeschrieben werden. Card/de la Rica (2006) schlagen eine Methode vor, um dieses Problem der sogenannten unbeobachteten Heterogenität auch in einem Querschnittsdatensatz zu lösen: Sie fügen die durchschnittlichen Charakteristika der Beschäftigten eines Betriebs als zusätzliche Kovariate in der Lohngleichung hinzu.

Wenn die durchschnittlichen Charakteristika der Beschäftigten und die Wahr- auch von seinem Einfluss auf die Lohngestaltung am ehesten begünstigt werden? Mit Hilfe von Interaktionstermen zwischen dem Betriebsratsdummy und ausgewählten individuellen Charakteristika sowie Quantilsregressionen haben wir festgestellt, dass diese Annahme nicht zutrifft. Eher das Gegenteil: Im Gegensatz zu kollektiven Lohnvereinbarungen bewirkt das Engagement des Betriebsrates, dass der Lohnunterschied zwischen Männern und Frauen reduziert wird. Zudem profitieren insbesondere Beschäftigte mit Abitur, aber ohne eine abgeschlossene Ausbildung (dies ist allerdings nur eine sehr kleine Gruppe auf dem Arbeitsmarkt) (Tabelle 1, Spalte 5). Auch wenn wir den Lohnaufschlag von Betriebsräten nach Lohnquantilen aufteilen, also untersuchen, welchen Lohnaufschlag Beschäftigte erhalten, die relativ wenig verdienen, stellen wir fest, dass gerade diese Beschäftigtengruppe von einem proportional höheren Lohnaufschlag profitiert. Betriebsräte unterstützen also vor allem Beschäftigte mit unterdurchschnittlichem Einkommen (Abbildung 1). Frauen mit geringen Einkommen profitieren somit am stärksten vom Wirken eines Betriebsrats. Beschäftigte im zweiten Perzentil der Lohnverteilung erhalten $14 \%$ mehr Lohn, wenn sie in Betrieben mit Betriebsräten arbeiten - der Lohnaufschlag liegt dabei im Durchschnitt bei $19 \%$, falls es sich um Frauen handelt, und bei 11 \% für Männer.

Interessant ist zudem die Frage, ob der Lohnaufschlag, der durch das Agieren der Betriebsräte erzielt wird, zum Lohnaufschlag durch Tarifverträge substitutiv oder komplementär ist. Die Antwort fällt für Tarifverträge auf Betriebs- und auf Sektorebene unterschiedlich aus. Die Interaktionsterme in Tabelle 1, Spalte 6 zeigen, dass sich in Betrieben mit einer Interessenvertretung das Lohnniveau weiter erhöht, wenn es einen Haustarifvertrag gibt. Dagegen wird das Lohnniveau von Tarifverträgen auf sektoraler Ebene nicht beeinflusst. Dies lässt die Schlussfolgerung zu, dass Betriebsräte ihre Mitbestimmungsmöglichkeiten durchaus zumindest indirekt bei betrieblichen Lohnverhandlungen für Lohnerhöhungen nutzen können, während ihr Einfluss auf sektoraler Ebene naturgemäß beschränkt ist. 

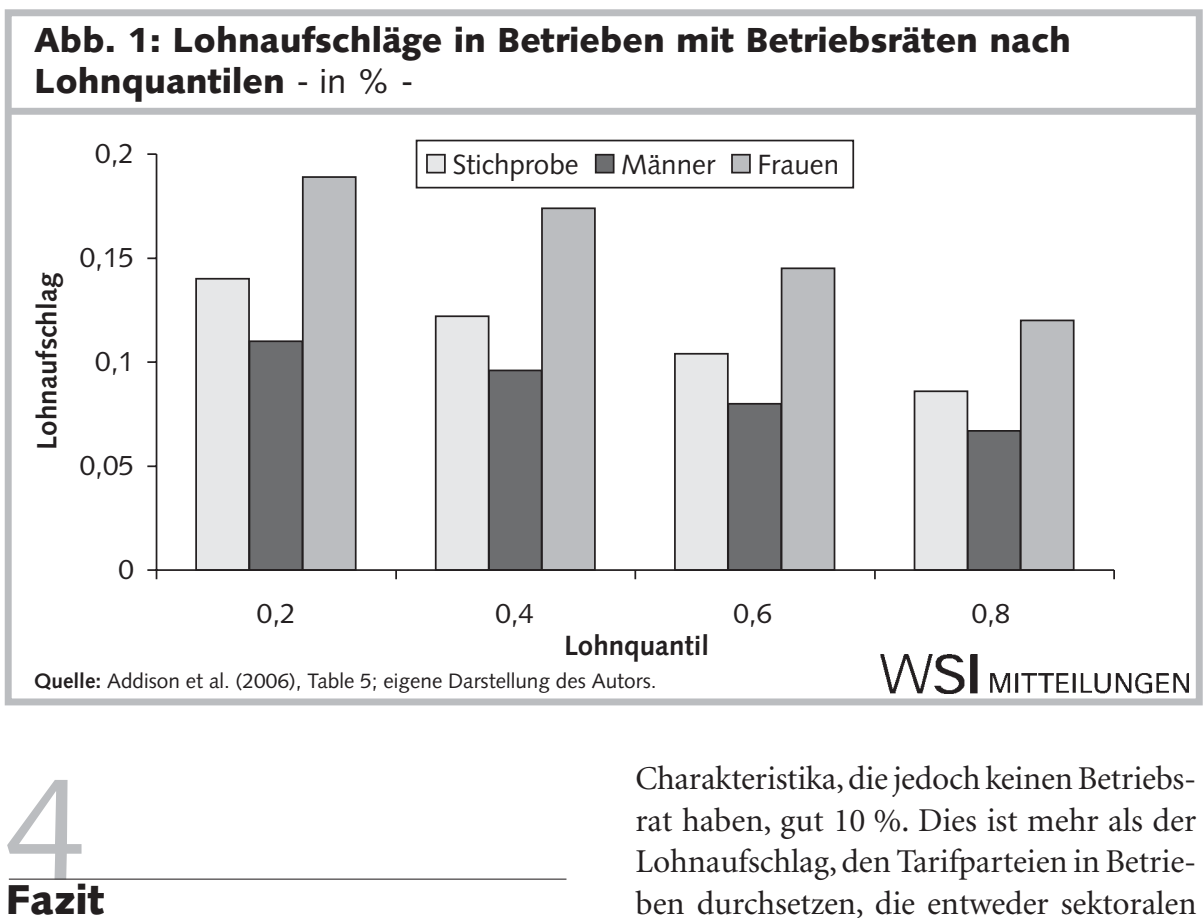

Wir können festhalten: Der Lohnaufschlag in Betrieben mit Betriebsrat beträgt im Vergleich zu Betrieben mit ähnlichen kann, was durch weitere Forschung zu spezifizieren wäre, auch ein Spiegel höherer Produktivität sein, die diese Betriebe aufweisen (Zwick 2004). Des Weiteren konnten wir zeigen, dass Betriebe mit Betriebsräten eine geringere Lohnspreizung aufweisen. Das heißt, der Lohnaufschlag, den Betriebsräte durch ihre Einflussmöglichkeiten auf die Lohngestaltung erzielen, ist für Bezieher relativ geringer Einkommen höher als für Mitarbeiter, die relativ gut verdienen. Somit spielen Betriebsräte eine wichtige Rolle für die Höhe und Struktur der Löhne in Deutschland, die bisher kaum beleuchtet wurde.

Eine zentrale Frage für weitere Forschungen ist nun, ob die höheren Löhne in Betrieben mit Betriebsräten eine Konsequenz einer stärkeren Umverteilung von Gewinnen an die Mitarbeiter sind, oder ob sie für ihre höhere Produktivität kompensiert werden. In letztgenanntem Fall würden die Mitarbeiter in Betrieben mit Betriebsräten keine Renten abschöpfen, obwohl sie mehr verdienen als ihre Kollegen in Betrieben ohne Betriebsräte.

\section{LITERATUR}

Addison, J./Kraft, K./Wagner, J. (1993): German Works Councils and Firm Performance, in: Kaufman, B./Kleiner, M. (Hrsg.): Employee Representation: Alternatives and Future Directions, Madison, S. 305-336 Addison, J./Schnabel, C./Wagner, J. (2001): Works Councils in Germany: Their Effects on Firm Performance, Oxford Economic Papers 53 (October), S. 659-694

Addison, J./Teixeira, P./Zwick, Th. (2006): Works Councils and the Anatomy of Wages, ZEW Discussion Paper 06-086, Mannheim Card, D./de la Rica, S. (2006): The Effects of Firm-Level Contracts on the Structure of Wages: Evidence from Matched Employer-Employee Data, Industrial and Labor Relations Review 59 (July), S. 573-592

FitzRoy, F./Kraft, K. (1985): Unionization, Wages and Efficiency Theories and Evidence from the U. S. and West Germany, Kyklos 38 (4), S. 537-554

Gartner, H. (2005): The Imputation of Wages Above the Contribution

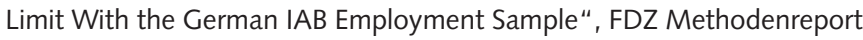
2, Nürnberg

Gürtzgen, N. (2006): The Effect of Firm- and Industry-Level Contracts on Wages - Evidence from Longitudinal Linked Employer-Employee Data, ZEW Discussion Paper 06-082, Mannheim
Heckman, J. (1976): The Common Structure of Statistical Models of Truncation, Sample Selection and Limited Dependent Variables and a Simple Estimator for Such Models. Annals of Economic Social Measurement 5, S. 475-492

Hübler, O./Jirjahn, U. (2003): Works Councils and Collective Bargaining in Germany: The Impact on Productivity and Wages, Scottish Journal of Political Economy 50 (September), S. 471-491

Kölling, A./Schnabel, C./Wagner, J. (2005): Establishment Age and Wages: Evidence from German Linked Employer-Employee Data, in: Bellmann, L./Hübler, O./Meyer, W./Stephan, G. (Hrsg.): Institutionen, Löhne und Beschäftigung, Nürnberg, S. 81-99

Stephan, G./Gerlach, K. (2005): Wage Settlements and Wage Setting: Results from a Multi-Level Model, Applied Economics 37 (November), S. 2297-2306

Wassermann, W./Rudolph, W. (2005): Betriebsräte nach der Reform, Münster

Zwick, Th. (2004): Employee participation and productivity, Labour Economics 11 (Dezember), S. 715-740 